\title{
WestVirginiaUniversity
}

THE RESEARCH REPOSITORY @ WVU

Graduate Theses, Dissertations, and Problem Reports

2006

\section{Coordinated traffic signal systems for municipalities}

Scott K. Nale

West Virginia University

Follow this and additional works at: https://researchrepository.wvu.edu/etd

\section{Recommended Citation}

Nale, Scott K., "Coordinated traffic signal systems for municipalities" (2006). Graduate Theses,

Dissertations, and Problem Reports. 1739.

https://researchrepository.wvu.edu/etd/1739

This Thesis is protected by copyright and/or related rights. It has been brought to you by the The Research Repository @ WVU with permission from the rights-holder(s). You are free to use this Thesis in any way that is permitted by the copyright and related rights legislation that applies to your use. For other uses you must obtain permission from the rights-holder(s) directly, unless additional rights are indicated by a Creative Commons license in the record and/ or on the work itself. This Thesis has been accepted for inclusion in WVU Graduate Theses, Dissertations, and Problem Reports collection by an authorized administrator of The Research Repository @ WVU. For more information, please contact researchrepository@mail.wvu.edu. 
Coordinated Traffic Signal Systems for Municipalities

\author{
Scott K. Nale
}

\author{
Thesis submitted to the \\ College of Engineering and Mineral Resources \\ at West Virginia University \\ in partial fulfillment of the requirements \\ for the degree of
}

\author{
Master of Science \\ in \\ Civil Engineering
}

L. James French III, Ph. D., Chair

David Martinelli, Ph. D.

Ronald Eck, Ph. D.

Department of Civil and Environmental Engineering

\author{
Morgantown, West Virginia \\ 2006
}

Keywords: Traffic Signal, Coordination, Maintenance, Operations

Copyright 2006 Scott K. Nale 


\title{
ABSTRACT \\ Coordinated Traffic Signal Systems for Municipalities
}

\author{
Scott K. Nale
}

Many municipalities have a need for a document which examines installation and operations methods used in coordinated traffic signal systems. Included within this document are the results of surveys of various municipalities which range in population from thirty three thousand persons to in excess of five hundred thousand persons. Along with the state of practice information, some conclusions and recommendations are defined so that municipalities may begin to implement coordinated traffic signal systems within their jurisdiction. This document will be of interest to those persons who manage, operate, and maintain traffic signal systems; in addition, engineers and municipal officials tasked with developing such a system will find benefit in this document. 
TABLE OF CONTENTS

TABLE OF CONTENTS.............................................................ii

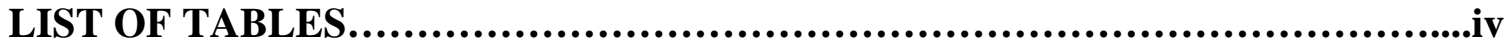

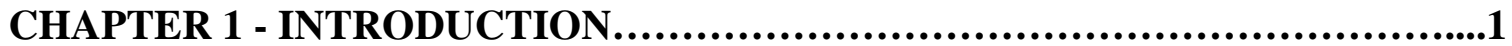

$\begin{array}{ll}1.0 \text { Introduction } & 1\end{array}$

1.1 Problem Statement 1

1.2 Research Objectives 2

1.3 Organization of Report 3

CHAPTER 2 - BACKGROUND...................................................................................... 4

2.0 Introduction 4

2.1 Signal Coordination 4

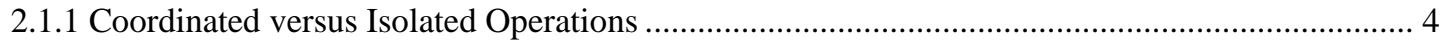

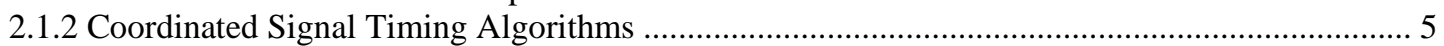

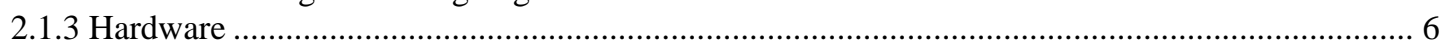

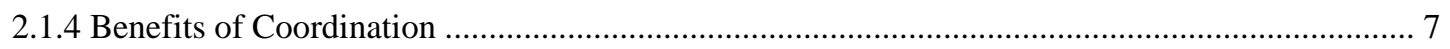

2.1.5 Time Based Coordination versus Interconnection .................................................................. 8

2.2 Interconnectivity in Traffic Signal Systems 9

2.2.1 Centralized versus Decentralized Communication ......................................................................... 9

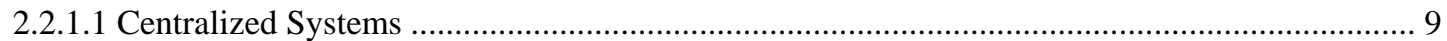

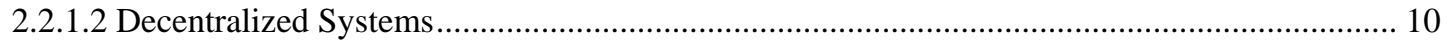

2.2.2 System Components ………………………………………………………………………… 10

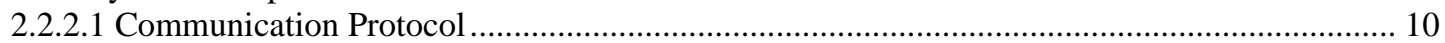

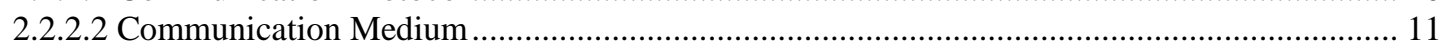

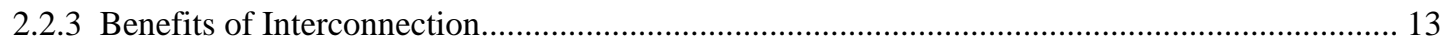

2.2.4 Concerns / Issues with Interconnection ........................................................................... 14

2.3 Matching of Human Resources with Potential Interconnectivity Benefits 14

$\begin{array}{ll}2.4 \text { Maintenance Practices } & 15\end{array}$

2.5 Concluding Remarks $\quad 15$

CHAPTER 3 - METHODOLOGY.................................................................................. 16

$\begin{array}{ll}3.0 \text { Introduction } & 16\end{array}$

3.1 Development of Contact Locations $\quad 16$

3.2 Development of a List of Important Topics 18

3.3 Data Reduction and Analysis $\quad 19$

3.3.1 Facility Stratification …………………………………………………………………………... 19

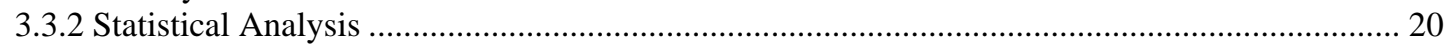

3.4 Concluding Remarks 20

CHAPTER 4 - RESULTS............................................................................................. 21

4.0 Introduction $\quad 21$

4.1 Low Involvement Municipalities 21

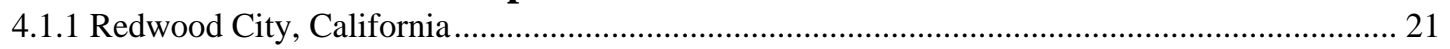

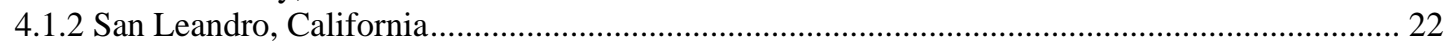

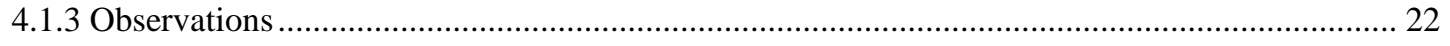

4.2 Medium Involvement Municipalities 23

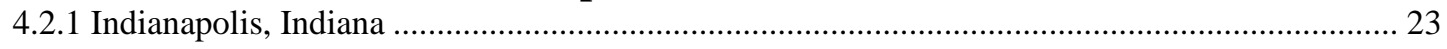

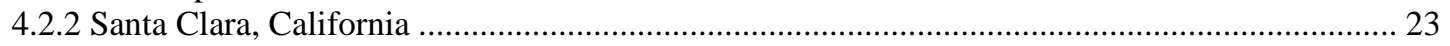

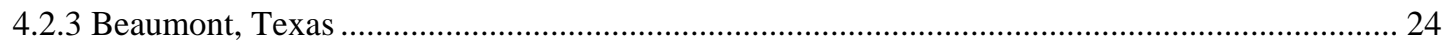

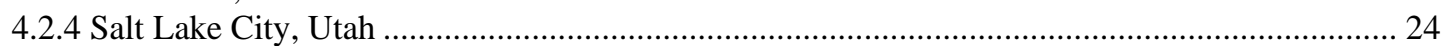




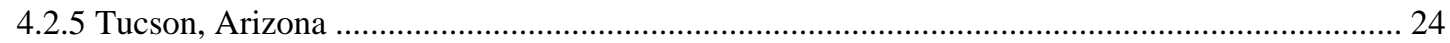

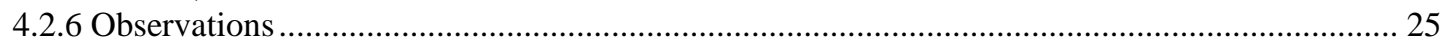

$\begin{array}{ll}\text { 4.3 High Involvement Systems } & 25\end{array}$

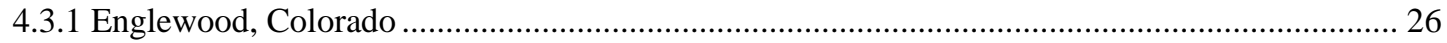

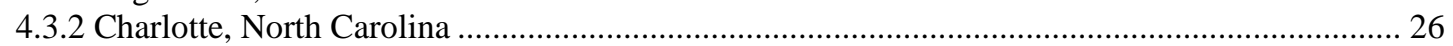

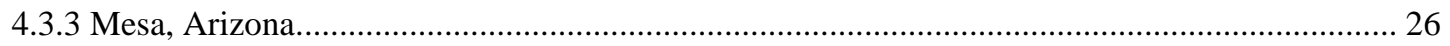

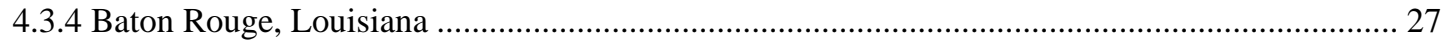

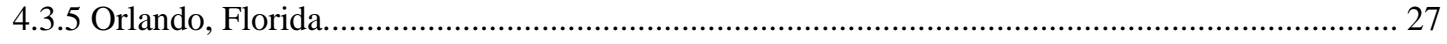

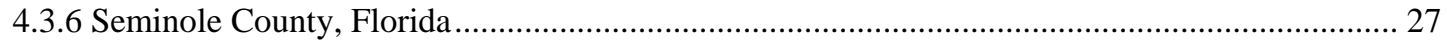

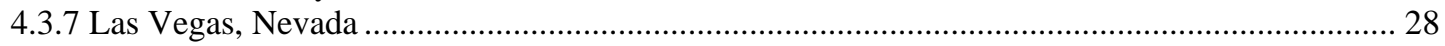

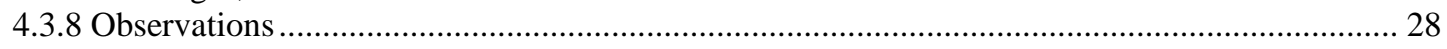

4.4 Signals Managed Per Workstation 28

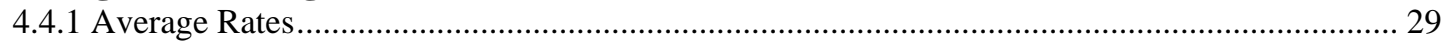

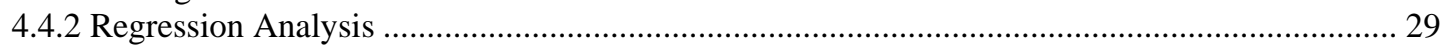

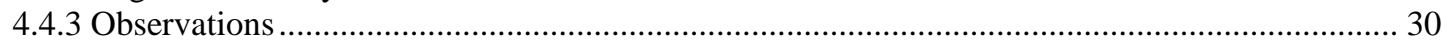

$\begin{array}{ll}4.5 \text { Staffing } & 30\end{array}$

4.6 Research and Planning $\quad 32$

4.7 Location and Layout $\quad 32$

4.8 Concluding Remarks $\quad 33$

CHAPTER 5 - CONCLUSIONS AND RECOMMENDATIONS .......................... 34

$\begin{array}{lr}5.0 \text { Introduction } & 34\end{array}$

$\begin{array}{ll}5.1 \text { Conclusions } & 34\end{array}$

5.2 Limitations of the Research 35

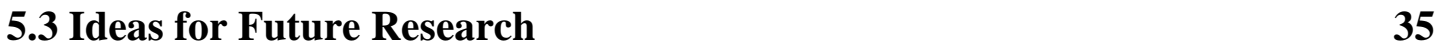

References ....................................................................................................... 37

Appendices.............................................................................................................. 38

Appendix A - Phone Interview Question List ................................................ A-1

Appendix B - Municipality Database Statistical Analysis.............................B-1

\section{LIST OF TABLES}

Table 2.1 Signal Timing Variable Definitions $\_5$

Table 2.2 Crash Experience Before and After Coordinated Signal Control___ 8

Table 2.3 Communication Media Costs and Speeds ___ 13

Table 3.1 List of Municipalities Contacted__ 17

Table 4.1 Staffing Conditions of Study Facilities__ 31 


\section{CHAPTER 1 - INTRODUCTION}

\subsection{Introduction}

Traffic signal coordination refers to a process by which traffic signals can operate in conjunction with each other (Garber and Hoel, 1999). This can be as simple as two signals operating with coordinated timing sequences or as robust as a series of signals along a busy arterial running in coordination to help provide higher efficiency across the corridor. While the applications of this process may vary by locality, several of the main concepts are applied universally.

Traffic signal management is becoming more important as a result of an increase of traffic in urban areas combined with a reduction in the rate of highway construction. The goal of traffic signal management is to better control traffic flows on the existing system in order to maximize capacity. Signal management issues include hardware components, coordination of the signal timings, traffic data compilation, incident management, and real-time intervention.

The best way to optimize the traffic signal system infrastructure is through the use of integrated equipment on a communications network (Gordon, et al., 1996). Such systems can provide the operators with data pertaining to traffic volume, hardware status, cycle timing, and other flow variables. A system, as the term is being used, refers to a combination off all components used operate the signalized intersections; these may include signal hardware, communication backbone, and software packages. The system can provide these data as they occur, and allow the traffic control personnel to intervene with signal timing changes in real-time, which results in a better response to traffic and congestion. These systems are also capable of informing the traffic control center, or the signal location from which the signals are managed, of incident occurrence and hardware failure at the moment it is detected.

\subsection{Problem Statement}

Currently, there are several commercially available software packages which can be implemented to provide for centralized traffic signal management. Some vendors have multiple software packages, with varying levels of capabilities and sophistication. While there is a wealth of information available on the capabilities of these packages, it is somewhat scattered, and has not been synthesized in one comprehensive document. Packages are described by each vendor in promotion literature, but this must be obtained from the respective vendors. Small urban areas generally lack the expertise and seldom have the means to perform a comprehensive survey of what is available. If the municipality cannot do this, then it is not likely that they can fully determine their needs for traffic signal management. This will deter the ability to then match these needs to the appropriate package. Finally, with the above deficiencies it can be difficult for these municipalities to anticipate the human resources and hardware infrastructure necessary to 
support the system. It would be beneficial to decision makers in small urban areas to have some background related to the implementation of these systems.

Currently, there is no comprehensive reference offering suggestions for the implementation of traffic signal management systems. The systems to which this discussion refers are the equipment and software necessary to operate, maintain, monitor, and adjust the traffic signal installations. Objective information describing the selection of the appropriate level of sophistication in the system and the human and technological resources to support the system is needed. It is the purpose of this research to conduct several case studies illustrating how municipalities implement and operate their system. In addition to providing the information from the case studies it is also the purpose of this report to provide some background for municipalities seeking to implement this sort of system.

\subsection{Research Objectives}

The overall goal of this project was to develop background for the implementation and operation of traffic signal management systems in municipalities. This goal was achieved through the following objectives.

1) Provide background pertaining to traffic control systems, signal coordination, and current traffic signal management strategies.

2) Identify a sample of traffic signal management software packages.

3) Contact vendors of the sample of traffic signal management systems and identify the abilities and limitations of their system and in addition, determine cities where these systems have been implemented

4) Survey a sample of municipalities which currently operate such systems and determine:

a. The manner in which the system is utilized

b. Human resources dedicated to these operations

c. Technological resources applied to these applications

5) Summarize the findings so that the information may be used to provide background for municipalities relative to:

a. The selection of a system with the appropriate functionality and requirement of resources

b. Limited technical guidance on the human and technological resources that will be needed to implement and operate these systems

c. Limited technical guidance on systems operations and selection

Study findings will provide for a better understanding of the many ways in which these systems are used. Also, with this information it will be possible to make a 
determination of what the appropriate plan of action is for implementation and operations.

\subsection{Organization of Report}

This report is divided into 5 chapters. Chapter 1 introduced the problem and outlined project objectives. Chapter 2 will provide background pertaining to traffic signal coordination and traffic signal management. Chapter 3 will describe the methodology used in the completion of this research. Chapter 4 will present the results of the research effort. Chapter 5 concludes the report with a summary of the primary conclusions and recommendations, limitations of the research, and suggestions for further research in the traffic signal management area. 


\section{CHAPTER 2 - BACKGROUND}

\subsection{Introduction}

This chapter will explore important background information related to traffic signal systems. A traffic signal system is defined as a combination of any number of traffic signals of which the operations is examined as a unit. It is recognized that traffic signal systems can have two major components: the signal coordination and the hardware interconnectivity. It is possible to have signal timing coordinated at adjacent intersections without any communication between them. Furthermore, communications between adjacent signalized intersections and between signalized intersections and traffic control centers even if the signal timings at adjacent intersections are not coordinated can often be beneficial. Therefore, these topics will be treated separately in this discussion, with Section 2.1 being devoted to signal coordination topics, and Section 2.2 being devoted to hardware interconnection. A third important issue of traffic signal management is the human resources devoted to the system. While traffic signal systems can provide a large menu of valuable tools and capabilities, their potential can only be realized if operated by a staff of adequate size and training. Section 2.3 is devoted to these issues.

\subsection{Signal Coordination}

\subsubsection{Coordinated versus Isolated Operations}

When it comes to the issue of signal operations, there are two in ways which signals can be operated. These are isolated and coordinated. Each approach has certain conditions under which each configuration should be used.

The simplest configuration is the isolated or "stand-alone" intersection. When an intersection operates in isolation, typically the traffic signal is fully actuated. Thus, only the vehicular demand within a short distance of the stop bar is considered in allocating the green time to the various phases. This greatly simplifies the operations at the signal, and may result in very efficient demand service in some locations. These intersections can be designed so that they have an appropriate level of responsiveness to traffic demands, which will assist in keeping the signal working at the desired state of efficiency and safety. Some signalized intersections can employ "volume-density" control, which is utilized on high-speed approaches to minimize the number of drivers shown the yellow indication a short distance from the stop bar. More basic signalized intersections employ "basic actuated" control that simply serves the standing queues and traffic arriving during the green (Gordon, et al., 1996). This approach is best suited to signals which are spatially separate from other signals; a spacing of one-half mile is commonly used but it is entirely dependent on field situations. For corridors that do not have a high level of vehicle platooning, and have somewhat more random vehicle arrival patterns, isolated 
operations are usually the most appropriate. However, at locations where the signals are very close or the queues from one signal may affect another, traffic operations can be improved if the signals operate under a coordinated configuration.

The second approach to operations is to coordinate the signal timings of adjacent signals. Adjacent signalized intersections are coordinated because of an understanding that the traffic platoons can flow through a series of traffic signals more efficiently than if they were dispersed, and the signal timings are coordinated to provide this flow (Skehan, 1996). In the case of coordination, the management of traffic streams is handled entirely differently than in isolated operations. The emphasis shifts from individual vehicles on the immediate approaches of each isolated intersection, to platoons of vehicles that should be progressed through an entire series of traffic signals with minimal stops. Effort is made to pass a platoon of vehicles completely through the network by timing traffic signals so that the platoons arrive at each signal during the green indication, thus, reducing wasted stop and start time. This is done by coordinating several key variables which can be found in Table 2.1 (Skehan, 1996). Through the proper timing of these variables, it is possible to maintain platoons of moving traffic.

Table 2.1 Signal Timing Variable Definitions

\begin{tabular}{|l|l|}
\hline \multicolumn{1}{|c|}{ Variable } & \multicolumn{1}{c|}{ Definition } \\
\hline Cycle Length & $\begin{array}{l}\text { The time required for 1 complete sequence of signal intervals } \\
\text { (phases). }\end{array}$ \\
\hline Split & $\begin{array}{l}\text { The percentage of a cycle length allocated to the green time of each } \\
\text { of the various phases in a signal cycle. }\end{array}$ \\
\hline Offset & $\begin{array}{l}\text { The time difference (in seconds or in percent of the cycle length) } \\
\text { between the start of green indication at each intersection as related } \\
\text { to the system time reference point. }\end{array}$ \\
\hline
\end{tabular}

In coordinated signal systems, the cycle length of all signalized intersections must be identical, otherwise, the cycles will not be able to remain in time synchronization with each other. The splits are adjusted at each intersection so that each individual intersection operates efficiently and without excessively delay. The offsets are adjusted at each intersection so that the green times on the coordinated corridor minimize the stoppage of platoons. The offsets between adjacent intersections are based on the travel time between the intersection and the size of the platoons. Ideally, the offsets can be set so that the platoons arrive at each intersection at the beginning of green.

\subsubsection{Coordinated Signal Timing Algorithms}

As signal hardware has evolved with technological advances, one improvement is occurring in the area of possible timing algorithms. At the earliest traffic signal installations, it was possible to operate only a single timing plan that would have to serve the entire daily demand with all its various fluctuations. The user would be required to 
select a single plan based on investigation of the historic traffic demand, but in fact, it is likely that it would operate below optimum efficiency most of the day.

As technology in this area has advanced, traffic signal designers are able to program multiple phase plans that are activated at certain times of day. This improved the efficiency of the intersections, as it was now possible to serve varied demands as they occurred. The various plans were initiated at pre-specified times of the day and week. This was enabled once the signal controller was capable of keeping track of the date and time, along with the memory to store the various plans (Gordon, et al., 1996). These plans were based on a consistent flow pattern that is derived from historical data of the corridor. The signal controllers, however, also had limitations in that only a finite number of phase timings could be stored, which meant that some demands were still not matched with optimizing plans. Furthermore, depending on the variability of the traffic throughout the day and week, the need for data and signal timing analysis could become overbearing. Finally, when prevailing flows varied from historical trends, inefficiencies could be encountered.

Another advancement in the technology involved the integration of demand sensing hardware, also known as actuation. The most common manifestation of the sensing technology is the use of induction loops placed in the roadway (Garber and Hoel, 1999). These loops in combination with a responsive signal controller are capable of modifying the timings, allowing a higher efficiency to exist at the intersection. In coordinated systems, individual signalized intersections are typically only semi-actuated. Side roads are actuated and the mainline receives a fixed window of green time plus any additional time left over from the side roads and other minor movements. The semiactuated systems also have the ability to employ various predetermined plans by the time of day and week. However, the shortcomings with these plans include long delays on side roads and the unconditional allocation of green time to the mainline, regardless of whether the time was needed. As such, there is still room for improved responsiveness to traffic flow. These semi-actuated systems are also subject to loop failures, which can be quite frequent depending on climate.

Further advancements led to traffic responsive systems. These are signal systems in which the cycle, splits, and offsets can be modified in real-time in response to traffic conditions. These systems have the potential to provide the most flexibility to meet prevailing traffic conditions. However, they have proven to be difficult to design, and during the transitions between plans, disruptions to traffic can occur. This is particularly troublesome if the system is too overly sensitive to traffic fluctuations. As such, the entirely responsive signals configurations have not received the level of wide spread acceptance that is consistent with their potential (Garber and Hoel, 1999).

\subsubsection{Hardware}

The most basic hardware involved in signal coordination is the controller. Each intersection has a local controller that responds to detector inputs related to demand and runs a specified algorithm to allocate time to the various green, yellow, and red 
indications. This local controller also monitors the time and can communicate with a master controller and central system software package.

In certain communication hierarchies, a master controller is located in the field to serve as the time keeper for a group of signals, and to communicate with the central system software package. The signal communications structure will be discussed in greater detail in Section 2.2.1.

The central system software package has many duties, most of which are not directly related to coordination, and thus will be discussed in Section 2.2. However, with respect to coordination, the central system software package can serve as a storage facility for timing plans that might be sent to the field by a technician. The software package is also a tool from which new traffic signal plans can be developed. In certain communication hierarchies, this software is also the keeper of the clock for synchronization purposes.

\subsubsection{Benefits of Coordination}

There are many documented advantages of coordinated signal systems. A citywide reduction of travel time can be experienced as a result of better intersection management (Lui, et al., 2002). Inefficient application of green time can be observed when the signal is showing green to a phase that has no queue to service. This often manifests itself in locations where upstream metering is occurring. This metering results in traffic being held upstream and not being able to use the green time being shown. However, through the use of timing plans that fluctuate with traffic demands, a signal is better able to serve the traffic volume that is current and not the volume that was or will be present at a peak during the day.

An additional benefit of coordinated signal systems is a reduction in certain types of accidents. "In corridors where a high number of accidents occur that traditionally can be attributed to coordination, the rear end percentage drop is fairly consistent" (Adams and Huffine, 1995). Table 2.2 presents accident frequencies at the intersections studied by Adams and Huffine (1995) before and after coordinated signal control. It should be noted that the study was conducted for one year before and after the signal changes. Not accounted for in this study were possible demand changes as a result of environmental factors or the allowance of an adjustment period for drivers to adapt to the new conditions. If these factors were considered, it is likely that values would differ. It should also be noted that the total number of persons injured after the implementation was lower that the total number injured prior to the change. 
Table 2.2 Crash Experience Before and After Coordinated Signal Control

(Source: Adams and Huffine, 1995)

\begin{tabular}{|l|c|c|}
\hline \multicolumn{1}{|c|}{ Intersection } & $\begin{array}{c}\text { Accident Frequency Before } \\
\text { Integration }\end{array}$ & $\begin{array}{c}\text { Accident Frequency After } \\
\text { Integration }\end{array}$ \\
\hline CR 4 & 52 & 50 \\
\hline CR 3 & 65 & 60 \\
\hline CR 11 & 52 & 60 \\
\hline CR 97 & 54 & 54 \\
\hline CR 19 & 80 & 48 \\
\hline CR 16 & 60 & 51 \\
\hline E. Ridge Rd. (1) & 137 & 150 \\
\hline E Ridge Rd. (2) & 90 & 70 \\
\hline Hudson St & 20 & 21 \\
\hline Portland Ave & 5 & 6 \\
\hline NYS 104 & 307 & 290 \\
\hline
\end{tabular}

Other advantages included in the benefits of a coordinated system are reduction in fuel consumption, driver frustration, pollution, and wear and tear on users vehicles as a result of smoother traffic flows. While such installations increase the quality of operations for vehicular traffic there is also a benefit for pedestrian traffic. A pedestrian will find that a mid-block crossing is easier as a result of the vehicular platooning. The platoons will affect larger gaps between vehicular arrivals.

\subsubsection{Time Based Coordination versus Interconnection}

There are two main ways to achieve the required time relationship for coordination between adjacent signalized intersections. These are time-based systems and interconnected systems. Each of these approaches are described below.

The less reliable approach is the time-based system. In this application, controllers at adjacent intersections lack communications between them. They are programmed such that they achieve required offset, cycle length, and splits. This approach is most likely the least expensive way to achieve such a configuration since the costs of establishing a line of communication are not incurred. However, any errors, modifications, or disruptions to the cycle length can greatly disrupt the coordination. One common disruption occurs because the clocks "drift" to a certain extent, and do not remain synchronized with one another in the long term. Once a disruption occurs, it is possible to have traffic congestion that is worse than if no attempt to coordinate the signals had been made. (Gordon, et al, 1996) 
The other commonly used option is to coordinate with intercommunications. This is the most preferred way to implement a coordinated system. Through the use of a hardwire or wireless connection, it is possible for a master controller (decentralized) or centralized system software to control the timing of each signal in the corridor. This allows the master controller to eradicate any discrepancies in the coordination. The negative aspect of this approach is that there is an increased cost that appears in both the extra equipment and in the data communication infrastructure. Section 2.2 discusses the various aspects of interconnectivity as it relates to traffic signal systems. (Gordon, et al, February 1996)

\subsection{Interconnectivity in Traffic Signal Systems}

As noted in Section 2.1, the interconnection of traffic signal controllers at adjacent intersections with master controllers and / or central system software packages is largely beneficial in establishing coordinated signal timings. The interconnection also enables a number of other benefits in the overall management of a signal system. The background related to traffic signal system interconnectivity will be explored along with the benefits and costs that the system operators might incur.

\subsubsection{Centralized versus Decentralized Communication}

There are two main communication layouts that are used in signal system design; centralized and decentralized. In a centralized system, all connected signalized intersections communicate with one location either to receive or deliver data about the operations of a particular intersection or signal head (Gordon, et al., 1996). These data can be either complex, as in transmitting a new timing plan, or very simple, such as sending a signal to notify the central control center of the signal's operating status. In a decentralized signal system, there is no direct communication link between the local controllers at each signalized intersection and the central system. All local controllers at the individual signalized intersections communicate with a master controller that is also located in the field. Only this master controller then communicates with the central system. Each format is described in detail below.

\subsubsection{Centralized Systems}

The communication system format which links each local signal controller directly to the central system or traffic control center is referred to as a centralized system. In such configurations, information is transferred across some communications channel between the signal and a central location. In the more complex systems, this information may include traffic counts and signal plans. In the less sophisticated systems, these data may be a one directional signal to the central system indicating a signal is in proper working order, or it may be an indication to the signal to reset the system time for synchronization purposes. There are many different types of communications media that can be used to transmit these data and some common ones will be discussed in detail in Section 2.2.4 


\subsubsection{Decentralized Systems}

In a decentralized system, each local controller is connected to a master controller in the field. The master control keeps all of the local controllers in time synchronization and provides for all communications with the central software (Skehan, 1996).

One advantage of a decentralized system is that some of the traffic signal management intelligence stays at the street level. As such, failures at the central system do not have as much impact on operations as if the system were controlled entirely out of the central system. For example, a failure at the central system can cause a total loss of coordination in the field if the system is centralized. However, in a decentralized system, the operations in the field should be relatively unaffected if such a failure occurs.

\subsubsection{System Components}

There are two primary system components to traffic signal management systems. These are communications protocol and communications medium. There are a variety of communications media that may be used in interconnecting traffic signals. Concurrently, there are a number of communications protocols that are used to transmit data between controllers and central software packages. These two issues are related in that the type of communication media required depends in part on the operation of the system and the communications protocol needed. These issues are discussed in the following section.

\subsubsection{Communication Protocol}

The issue of communication protocol is a very important detail in relation to the hardware aspects of traffic signal systems. In the past, and to a large extent still today, each manufacturer developed their own proprietary format for the communication of data, otherwise known as a communication protocol (FHWA, June 2001). As a result, components from different manufacturers are most often not capable of communicating fully with other brands of hardware. Hardware incompatibility may be resolved in one of two ways. Either a signal system owner is required to obtain all of their equipment from a single supplier or a vendor must provide a software patch, which can translate the communications. Both of these solutions have drawbacks. The first approach causes the owner to become dependent on a single vendor, which inhibits competition and therefore competitive pricing. Also, this can stop a municipality from finding systems that provide the most desirable features and can leave them with an obsolete system if the vendor stops production. The patch option can result in a reduction of services or incomplete compatibility. These patches may or may not be completely reliable and frequently do not provide total support for some of the more complex features to be utilized. As a result, the application of software patches to facilitate communications between different types of signal equipment have limited success and are not considered as the most desirable operating condition. (Gordon, et al., 1996)

These situations have led to an increasing need for a standard communication

protocol. In response to this need, in 1996 the Federal Highway Administration (FHWA) 
asked representatives from the public and private sector to formally develop a set of communication standards for transportation management and monitoring devices in order to include signals and other hardware commonly called Intelligent Transportation Systems (ITS). These standards will provide for both "the rules for communicating (called protocols) and the vocabulary (called objects) necessary to allow electronic traffic control equipment from different manufacturers to operate with each other as a system." (NEMA, 1997) This standard would then need to be adopted by all manufacturers. The standard, titled the National Transportation Communication Information Protocol or NTCIP, will involve two separate parts: (1) the communications structure (which was adopted 2002) and (2) the vocabulary format (which to date has not been finalized). The communications section of the standard is being used to ensure that each component of these systems is capable of sending and receiving information in a recognized format. The vocabulary section will be used to ensure that each component in the system interprets the standard communication in a uniform way, allowing for each manufacturer's components to respond in the way each is intended to respond. Adopting these standards will grant significant freedom to those municipalities operating an NTCIP-compliant system. It will allow municipalities to purchase the system components from any compliant manufacturer to best suit their individual needs.

\subsubsection{Communication Medium}

A pivotal issue to consider is the communication medium, which is used to link the controllers to each other and to the control center. This decision is partially determined by the volume of information to be transferred between system components. Many of the constantly connected centralized communication systems need a medium capable of transferring large amounts of data. Assuming the systems use the NTCIP standard, which includes a large volume of data, a larger capacity communications medium is needed, whereas some of the systems which connect to the central system on a time-based system are capable of using lower data rate lines. The benefits and shortcomings of each type of media vary and should be analyzed in detail in the design step. There must be a balance determined between speed and cost. Fiber optic line, for instance, can transfer a large amount of data very quickly but the cost for such a system is much greater than other types of communications media.

The issue of communication medium selection is critical and site-specific. Variables involved in this selection can include: system type, system vendor, relative location, field conditions (such as line of sight between intersections) and cost. Discussed below are several of the more commonly implemented options for communications in either a centralized or decentralized configuration.

Twisted pair refers to a wire, consisting of multiple twisted strands, connected directly from point to point, most commonly from the local signal controller to the master controller from which it receives directions (White, 2002). While this is one of the lowest cost choices, it does have several drawbacks. Transmitting large amounts of data, such as entire signal databases, to and from the signal can be quite slow or even not possible due to the slow speed of data transmission (about 1/40 the speed of fiber optics). 
Another approach for communication is the use of short-wave radio. This medium has relative advantages in locations where a wire connection may be difficult to achieve due to signal placement or distance. Benefits are seen in the fact that a physical connection is not required, meaning there is no need to lease space on a utility pole, install new poles, or incur the cost of expensive trenching operations. While this medium appears attractive at the surface, there are also some serious drawbacks to this approach. This approach requires a line of sight between the antennas, which may be obstructed by buildings, vegetation, and terrain. Also, in locations with dense radio traffic, the data signal may be interrupted such that the transmission can be distorted or completely lost. The loss of this communication link can disrupt the functionality of the coordination.

To facilitate a connection from the field controllers to the central computer, many installations utilize an established system by transferring signal data through a telephone modem installed in the field controllers. This system has a significant advantage in terms of cost. The reason for the advantage is that this approach utilizes a sector of infrastructure, telephone lines, which are already in place. As with other systems, there are also some relative disadvantages to this approach. The main drawback is that if a constant connection is desired, it is difficult to obtain with this medium. This sort of connection is operated as such; the modem will dial the receiver of the data and begin the transfer. In the event that a system failure occurs and the controller cannot identify the disruption in a timely manner, the system may not detect the situation at all.

The final commonly applied medium to be discussed is fiber optic line. The advantages of this approach are the transfer rate and quality of the signal over a long transmission distance. The choice of fiber optic line is most suited to situations where a large amount of data is transferred. Fiber optic lines are best suited for large data streams due to the transfer rate of 10 Gigabytes per second, which is one of the fastest data transfer rates for this sort of communication. The largest single drawback is the cost of installing and operating a fiber optic system. Many municipalities have chosen to lease part of a system that is owned by others, which results in lower initial costs. It should be noted that if budget is a concern, the choice of fiber optic communications needs to be closely examined to determine the need for such a system.

Data included in Table 2.3 Communication Media Cost and Speeds was obtained from White, 2002. This table allows for a comparison of data media. Values of speed, Mbps, refer to megabytes per second and, Gbps, gigabytes per second. Cost values do not included the cost of signal amplification hardware, the actual transmission equipment or the installation of the medium. 
Table 2.3 Communication Media Costs and Speeds

\begin{tabular}{|l|l|l|l|}
\hline Medium & Data Rate & $\begin{array}{l}\text { Maximum } \\
\text { Distance }\end{array}$ & Cost (wire only) \\
\hline Fiber & $10 \mathrm{Gbps}$ & $100 \mathrm{miles}$ & $10.00 \$ /$ foot \\
\hline $\begin{array}{l}\text { Short Wave } \\
\text { Radio }\end{array}$ & $100 \mathrm{Mbps}$ & $20-30 \mathrm{miles}$ & $3,500 \$ /$ intersection \\
\hline Phone Line & $<2 \mathrm{Mbps}$ & $3-4 \mathrm{miles}$ & $1.50 \$ /$ foot \\
\hline Twisted Pair & $250 \mathrm{Mbps}$ & N/A & $3.00 \$ /$ foot \\
\hline
\end{tabular}

Gbps=Gigabytes of data per second; Mbps=Megabytes of data per second

Source: White, 2002 and current PennDOT Cost Data

\subsubsection{Benefits of Interconnection}

One beneficial feature of most of these systems is a standard connection to each signal, allowing the control personnel to know almost immediately after a signal has experienced a problem. This connection may be one that is constantly transmitting, one that occurs on a consistent schedule, or one that transmits in response to a problem. Many of these systems are able to notify the system managers of traffic data such as volumes and occupancy, performance measures such as green splits, operational issues such as a signal going into flash, and even security issues, like a controller door being opened. Through the rapid passing of this information, it is possible for system managers to mobilize whatever resources are needed to return the signal to normal operations. This enables the system to better prevent congestion throughout the network.

Some packages will even allow for incident recognition in real-time. These systems can monitor vehicle demand in multiple locations along a corridor. By observing a high demand at one section of a corridor and little or no demand at subsequent intersections it is possible to take note of an incident. With a large traffic back-up showing for a certain stretch of arterial and no demand past a certain point, the control personnel will be able to recognize that an incident has occurred and the approximate location of the incident. As a result of this sort of algorithm, the operators are better equipped to handle and clear the incident or fix faulty equipment in a timely manner. As a result of the quick response by emergency personnel, the corridor returns to normal operations in a more timely fashion.

These systems are also capable of recording the data for signal professionals to examine and develop new timing plans. Some of these systems are even capable of putting the data directly into programs such as SYNCHRO, which can be used to generate new timing plans for these intersections (Gordon, et al., 1996). 


\subsubsection{Concerns / Issues with Interconnection}

There are a few key issues to be noted in an interconnected system. First, is the infrastructure that must be implemented in order to best operate an interconnected system. Interconnection can cause high initial expenses or costly recurring investments depending on how the interconnection is achieved. In some instances, the signal system owners may install their own communication lines inside their available right-of-way. These installation costs can be substantial, particularly if the cable must be buried. In other instances, the owners may wish to rent a communications line, or rent space on a utility pole to attach the cable. Space on utility poles has become increasingly scarce and expensive in recent years as the change to broadband communications has required an increase in the number of data lines placed on utility poles. Often systems which are not centralized lack the robust requirements for data transfer as those systems that are centralized. In the decentralized systems, often the only information passing across a data line is the coordination of controller clocks. Finally, the design of the interconnectivity can be quite complex if media such as fiber optics are used, or if multiple media types are used.

\subsection{Matching of Human Resources with Potential Interconnectivity Benefits}

It should be noted that it is necessary to match the amount of staffing to the functions that are necessary to operate the system in the desired manner. The benefits of interconnectivity cannot be fully realized unless the traffic operations center staff has adequate time and training to carry out monitoring and management of tasks. There are several types of personnel which can be used to operate these types of systems. This information came from speaking with the municipalities polled but it is necessary to present this information at this time. These classifications are system manager, signal specialist, technician, and field technician. Note that because there are different titles it does not necessarily mean that one person cannot be trained to do all of these tasks.

A system manager is a person who is responsible for the management of both personnel and equipment on the system. Such a person would be capable of making decision regarding the operations of the system such as if and when to make timing adjustments. This person would also direct other personnel to make field adjustments such as correcting broken field equipment and would manage the day to day operations of the signal system.

A signal specialist would be someone who has experience in traffic signal timing plans and coordination timing plans. This person would likely be trained in traffic flow theory or transportation engineering. Such a person would be responsible for implementing and adjusting the traffic signal timing plans. Additionally, such a person would make adjustments to coordination plans in the event that a corridor was not operating to its fullest potential.

A technician would be a person who is familiar with the operations of the coordinated traffic signal system software and the communications network. This would 
likely be a person with a background in information technology and also training in the software package utilized in the municipality. Such a person would be responsible for maintaining the operations of the computers and communications of the system additional this person would interact with the software package to make adjustments as needed.

A field technician would be a person who is familiar with the hardware components of traffic signals. Such a person would likely have a background as an electrician with experience in traffic signal components. This person would be responsible for replacing traffic signal equipment in the field. Tasks such as rewiring signal heads and replacing signal bulbs in the field would be in the range of duties for this person.

\subsection{Maintenance Practices}

An additional important component of signal installations is maintenance. Maintenance is commonly divided into three types: responsive, preventative, and design modification. Responsive maintenance includes those practices which only go into effect once some failure has occurred in the system. Preventative refers to the practices in which system equipment is replaced and repaired to prevent a system failure. Finally the design modification component refers to practices related to the alteration of a system in order to provide more optimal operations (Kraft, et al., 1997).

According to research conducted by Walter Kraft, responsive maintenance is performed by all agencies and this task utilized fifty percent of an agencies budget. Preventive maintenance is performed by most organizations and this task comprises thirty three percent of the budget. Finally three quarters of organizations perform design modification activities and this task comprises seventeen percent of the budget (Kraft, et al., 1997).

Kraft also notes that on average municipalities employ approximately 2.6 persons per one hundred thousand people of population. This equates to thirty eight signals per employee (Kraft, et al., 1997).

\subsection{Concluding Remarks}

This chapter has explored the background related to the two main components of a traffic signal system, coordination and interconnectivity. This should be a valuable technology transfer resource for municipalities considering the installation of such a system. The remainder of the report describes the research methods and results, which are focused on the lessons learned from actual installations of these systems by other localities nationwide as conveyed by the system owner/operators. 


\section{CHAPTER 3 - METHODOLOGY}

\subsection{Introduction}

Objective information supporting decisions regarding the selection of the appropriate level of sophistication in the system and the human and technological resources to support the system is needed. It is the purpose of this research to conduct several case studies illustrating how municipalities implement and operate their systems. This information is then related to parameters of the municipality that describe its overall size and number of traffic signals. It is also the purpose of this research to outline a few of the more common traffic signal management packages so that the different levels of sophistication can be defined. To accomplish this task, a study had to be designed and completed so that a significant amount of data pertaining to these systems could be gathered, recorded and analyzed. In order to properly understand these practices, it was necessary to review the applicable technologies, the vendors who supply them, and the localities which implement these systems. Having obtained a better understanding of this background material, it was possible to determine the information that should be collected from those persons who maintain and operate traffic signal systems.

With the preceding overall goals in mind, the major steps of the research were as follows:

1) Identify a sample of vendors that develop and implement/install software and hardware used to manage multiple traffic signals.

2) Become familiar with these systems and locations in which they have been implemented.

3) Develop a survey to obtain information regarding system configuration in addition to the uses and resources for those individuals who operate these systems.

4) Develop a list of those individuals who operate these systems and contact them for discussion about their installations.

5) Compile the information obtained from individual operators in such a way that conclusions can be made relative to signal system capabilities and required human and technological resources.

6) Document the findings in a report that will provide background to signal system planners, municipal traffic officials, and signal system managers.

Each of the primary steps will be discussed in a section below.

\subsection{Development of Contact Locations}

A preliminary list of traffic signal management system installations was developed using several approaches. It should be noted that system installations were defined as those locations in which multiple signals were interconnected and a complex 
software system existed to interact with the signals. Through professional experience, it has been determined that many locations have two or three signals coordinated with a medium between them but without the complex software or connection to a centralized location. This configuration works well for isolated clusters of traffic signals outside of a heavily signalized corridor. These locations were not the type of systems that were examined in this research. It should be noted that the list is not a complete compilation and as a result does not cover all possible system configurations. The technical information was obtained from four major vendors of software and hardware which are used for traffic signal management. These vendors were Eagle, Econolite, Naztec, and U.S. Traffic. This information included jurisdictions where at least one of the systems was being operated. Those jurisdictions formed the first draft of the list of contacts. Each municipality was contacted in order to determine who was responsible for the traffic signals. As the surveys were conducted, the individual who was responsible for the operations of each location was asked if they knew of other signal system installations in operation, and those jurisdictions were then added to the list for the researcher to contact. Each municipality on the preliminary contact list was not contacted as this would bias the results to those vendors which provided the information, but by contacting additional municipalities a larger sample of suppliers could be analyzed. The list of municipalities contacted is contained in Table 3.1.

Table 3.1 List of Municipalities Contacted

\begin{tabular}{|l|l|r|}
\hline \multicolumn{1}{|c|}{ City } & \multicolumn{1}{c|}{ State } & $\begin{array}{r}\text { Population } \\
\text { (persons) }\end{array}$ \\
\hline San Leandro & California & 80,000 \\
\hline Charlotte & North Carolina & 541,000 \\
\hline Beaumont & Texas & 114,000 \\
\hline Tucson & Arizona & 487,000 \\
\hline Mesa & Arizona & 396,000 \\
\hline Orlando & Florida & 186,000 \\
\hline Santa Clara & California & 102,000 \\
\hline Seminole County & Florida & 365,000 \\
\hline Baton Rouge & Louisiana & 228,000 \\
\hline Indianapolis & Indiana & 782,000 \\
\hline Englewood & Colorado & 32,000 \\
\hline Las Vegas & Nevada & 115,000 \\
\hline Redwood City & California & 75,000 \\
\hline Pleasant Hill & California & 33,000 \\
\hline Salt Lake City & Utah & 182,000 \\
\hline
\end{tabular}

Census data obtained from the United States Census Bureau, based on year 2000 census. Population rounded to the nearest 1,000 people. 


\subsection{Development of a List of Important Topics}

It was determined that several general focus areas would need to be addressed. The successful determination of these areas was achieved through the surveying of system operators. These areas were determined to be resource allocation, system capabilities, system uses, and system type. The survey was conducted informally, through phone conversations with the individuals responsible for the traffic signal operations within a municipality. A formal questionnaire was not developed, resulting in the surveys taking on a conversational format, however a sample list of topics covered during each survey is included in Appendix A. The phone survey afforded distinct advantages over more rigid approaches such as mailed questionnaires. One benefit was this format allowed for the individuals being surveyed to stress the points they found to be most important while allowing the researchers to also focus on important ideas as they surfaced during the interview. Also, the phone survey allowed for names to be added in a timely manner to the contact list. Care was taken to ensure that the researcher and the person being surveyed were discussing the same issue, often defining items and restating questions to reach this goal. Additionally, the researcher had little to no prior information on the traffic signal management installation, which prevent personal biased from interfering in the process.

Prior to contacting the system operators, the decision was made that resource allocation would be the first area of focus. Resource allocation was determined to be the most important of the focus areas with respect to the project goal, as many of the small municipalities for which this research is being targeted do not have the resources to devote a large staff exclusively to traffic signal management. Some of the questions that were asked related to how many individuals were assigned to work on the system, how many signals were controlled or monitored, the quantity of and what sort of hardware was dedicated to the system.

The second area of interest was determined to be what the system was used for in the municipality. Different jurisdictions have different plans for the best use of the system capabilities. Some of these approaches are discussed in the analysis portion of this research. These questions focused on ascertaining information related specifically to staff members duties, staff interaction and access to the system, and the system capabilities that were utilized.

The third area of interest was system vendor and traffic signal management software type. The questions in this area addressed the design of the system. Variables such as centralized/decentralized control, communications medium, and number of signals were discussed. Also the discussions touched on the master configuration, if such a layout existed, along with some of the communication issues and how they were addressed. Examples of such issues are included in the municipality explanations.

In addition to the phone interviews, one of the signal system vendors contacted offered to provide a database of customers that included both the number of signalized intersections managed and the number of computers in the traffic management center. 
Both the vendor and customers are to remain anonymous, however, the database was valuable in providing an estimate of the relationship between the system size and the required technological resources. The database contained the information for 58 of their customers with population ranging from 3,510,000 to 2,000 persons living within the municipality. The analysis of the overall database is described in the following section.

\subsection{Data Reduction and Analysis}

\subsubsection{Facility Stratification}

After the information had been obtained, it was discovered that municipalities needed to be grouped due to the many different system configurations and variables that could be employed by municipalities. These variables could include things like frequency of communication with field equipment, level of interaction with the signal equipment, and progressive approaches to traffic control (such as merging traffic control with emergency service). Municipalities were broken into three different groups based on the operational configuration employed by each jurisdiction. Operational configuration referred to the system layout, personnel tasks, level of involvement, and functionality of the system.

- The first group was comprised of those municipalities which did not make use of the tools included in the signal infrastructure employed at their installation. An example of such a location would be one which had tools to conduct traffic data collection without field personnel but due to various reasons did not utilize this capability.

- The second group was comprised of those municipalities that used the system only for data collection or signal timing optimization but not both. An example of an installation which would be classified in this group would be a location where signal equipment was utilized to count vehicles but if any timing adjustments were to be implemented such changes would be completed in the field. The locations were determined to have only a marginal benefit from the existing equipment.

- The third group was comprised of those municipalities which used the system for both signal timing optimization and data collection. An example of this sort of location would be one which actively adjusted the signal timings at locations where problems existed or utilized components of the installation to collect data and reevaluate signal timings on a regular basis. The municipalities in this group were considered to be obtaining the maximum utility from their system.

After the groups were defined, each jurisdiction was analyzed individually to determine the routine practices and personnel requirements. Upon final inspection, there were some conclusions made about the state of practice within each group and how each could be improved. 
Three analyses were performed with the database of customer data provided by the signal system vendor. First, the data were used to determine the number of signals managed per workstation, or computer which can log into the system and manipulate any component of the signal equipment. The average, maximum, and minimum rates were identified, in addition to the standard deviation and coefficient of variation. Second, linear regression was applied to develop a linear model that provided the number of signals managed as the independent variable, and the number of workstations as the dependent variable. Lastly, a regression model was developed with the data assuming a logarithmic relationship. The coefficient of regression $\left(\mathrm{R}^{2}\right)$ is provided for each regression model so that the user can identify the statistical reliability of the model and judge its appropriateness for application.

\subsubsection{Statistical Analysis}

It was determined that the best way to analyze the data was to separate the municipalities into three groups. The boundaries between groups were made based on the extent to which the municipality implemented the potential capabilities of the given system. Municipalities that devoted little time or effort to the management of traffic signals through the system were considered to be "low involvement." Those locations which are categorized as low involvement did not interact with any component of the installation unless there was a signal which had experienced a failure or a complaint from the public had been made. These municipalities are discussed in Section 4.1. Likewise, those that increasingly implemented the potential advantages of their system were identified as "medium involvement" and "high involvement", which are discussed in Sections 4.2 and 4.3, respectively. In a medium involvement jurisdiction individuals either interacted with the system for data collection or signal optimization but did not actively implement both services while in a high involvement installation individuals both collect data and optimize signal timings at a regular interval. The frequency at which each location maintained those services determined if the system was a medium or high involvement. Section 4.4 concludes the chapter with a description of the statistical analysis of the customer database provided by one of the signal system vendors.

\subsection{Concluding Remarks}

This chapter has outlined the important steps carried out in the conducting of the coordinated signal system research. Chapter 4 contains a detailed description of each of the systems identified. The descriptions are presented according to the grouping based on activity level that was defined earlier in this chapter. It also contains the results for the statistical analysis of the customer database. Chapter 5 contains a summarization of the experiences of the surveyed municipalities and the research, as well as the limitations of this research and recommendations for further research in this area. 


\section{CHAPTER 4 - RESULTS}

\subsection{Introduction}

Below is a brief write up of the jurisdictions which were contacted and the information provided by individuals at each location. The discussion by location is followed up by some discussions on signals per workstation and staffing.

\subsection{Low Involvement Municipalities}

Two of the municipalities contacted fall into the low involvement municipalities grouping. These municipalities make minimal or no use of the capabilities of the coordinated signal system. Based on experience there are many municipalities that fall into the low involvement category, however, identifying such municipalities was difficult due to minimal or no information existing on these installations.

\subsubsection{Redwood City, California}

The Redwood City system controlled forty-five intersections across a twisted pair system. The main function of the system is to keep all the signals timed consistently. The system is also used to store information about system status changes. The central computer is located in the traffic office with little or no interaction from the staff. This system is not located in any centralized traffic control center and there are no personnel who are responsible for the daily operations of the system. Interaction with the system occurs only during instances such as a hardware failure being reported or new timing plans are to be loaded. Otherwise, the system runs without any monitoring or interaction from staff.

Redwood City runs forty-five of the sixty signalized intersections on this system and has plans to add another fifteen in the near future. This entire system is staffed by one individual who has duties other than the operations of traffic signals. One issue noted by the staff in Redwood City is that the signals are connected by twisted pair lines, meaning that only four signals may be run on one communication line. This has become an issue as the city integrates additional signals. The lack of additional communication capacity has made it difficult to add the additional signals. As a result, each new cluster of signals added requires a new line run from the central office to the field.

The main advantage to this approach is that little to no human resources are involved in the operation of this system on a regular basis. This practice frees up municipality resources while still providing consistent and coordinated progress across the city's main corridors. 


\subsubsection{San Leandro, California}

San Leandro originally designed their system to operate twenty of the fifty-two intersections operated by the municipality. The system was designed as a series of signals connected to the central computer across a fiber optic communication backbone. At construction, the signals were installed and connected to a local fiber optic service provider that furnished the fiber connections in the area. The city, in turn, leased the space from the utility company. Some time after the system was in place and operating, the fiber optic service provider company declared bankruptcy and ceased service. This left the city with only four of the original signals still connected to the system.

The city is currently looking for ways to regain operability to the signals. The city continues to maintain the system for the remaining signals. When a complaint is received regarding the signals still connected to the system, the system is used to examine and correct the problem. The staff provided by the city consists of four individuals who are responsible for all the signs, signals, and intersection designs for the city. These people use the system for the implementation of new timing plans at the four connected signals. This location was classified in the low involvement group due to circumstances beyond the control of officials in the city.

\subsubsection{Observations}

When a municipality is considering the installation of a centralized signal system, it is important that the operations procedure, or a clear plan for the utilization of the system, be determined during the initial planning. Variables such as system uses and staffing should be determined when the system is still in planning so that adequate resources may be allocated to the project. By determining how much data the system will be expected to transfer, it will be possible to determine the type of communications backbone that should be employed. Detailed planning will prevent extensive costs in the future for adding additional lines of communication.

Staffing of the system should also be determined prior to installation. If it is determined that a system will be set up to handle most of the day-to-day activity automatically, then a small staff with various other duties can handle system operation in addition to their other duties. In fact, the system can be used to reduce the amount of time that these people must invest on the signals which are connected, allowing them to access many signals from one location when the need arises. In this way, the system can in effect offset its cost by saving work for the employees.

Also during system planning, a secondary data connection should be planned for, in the event that something happens to the main communication backbone. While the secondary system may not need to be installed during the initial installation, having backup communications via dial-up modems or short wave radio may be a good way to proceed if the communication system is not to be owned by the municipality or governing highway department. This planning can result in the smooth integration of a system into a small municipality resulting in a quality system for many years into the future. 
However, it is recognized that this is a luxury that most municipalities may be unable to afford.

\subsection{Medium Involvement Municipalities}

Medium involvement municipalities are those that are either marginally proactive towards both the system data collection and coordination capabilities or are very proactive in either one or the other.

\subsubsection{Indianapolis, Indiana}

Indianapolis maintains two separate systems for traffic signal control and data collection. There are five individuals on staff who maintain the hardware and equipment for the city. These people are employed by the city to provide overall maintenance duties, and are not specialists in signal maintenance. These persons repair system hardware components as the need arises while also replacing signs and handling other duties around the city. The person who most affects the operations of the signal systems is a private consultant who makes adjustments to the systems as needed. In the event of a hardware failure, the consultant contacts the maintenance department and they make the necessary repairs. The consultant is also responsible for addressing congestion and poor coordination though the systems. Both systems were put in place to help reduce pollution and delay within the city.

The layout of the system consists of one robust system in the CBD capable of real-time data collection and a smaller system running the outlying signals to provide coordination in the outer areas of the city. The robust inner system is used for control and data collection, while the outer system functions only for coordination. The general approach to signal timing is to not alter the timing unless there is a serious problem.

It should be noted that this location has overcome the common problem of finding experienced technicians to work in the traffic control center by procuring the expertise of a consultant on an as needed basis.

\subsubsection{Santa Clara, California}

This location is currently running an antiquated system that is no longer supported by the company that produced it. However, it is still providing the capabilities and utility for which it was installed. This system is used to develop coordination and timing plans that can then be downloaded from the office to the master controllers in the field. This system is used to control 115 of the 120 signals within the city’s jurisdiction.

This jurisdiction also has a new system in operation that has not been wellreceived by the staff as of yet. According to the manager of the system, the newer system is set up in a manner that is hard to navigate. It was indicated that the staff had not received much training with the new system which is the probable cause that it is difficult for the staff to use. 
There are two system managers who do the timing plans, coordination plan modifications, and monitor the system. It was noted that in the opinion of the manager, the traffic control center is currently undermanned. At the time of the interview, the supervisor was hoping to add as many as three more persons to the staff.

\subsubsection{Beaumont, Texas}

Beaumont's system is comprised of seventy-five signals, all connected to master controllers, which are in turn connected to the traffic control center via a phone modem connection. The system personnel include two supervisors and three technicians. These people handle repairs, trouble calls, and preventative maintenance. Also involved in the operations is the city traffic engineer who does all the signal timings with the system and a few system technicians who go into the field to program the timing plans into the master controller.

It should be noted that at the time of the telephone contact, the signal system was new and the operators were still learning the functions. Hence, the staff was hesitant to work with or alter the system. None of the data collection or retiming features were being used, but it is anticipated that these features will be utilized in the future. At the time of the interview, the only feature being utilized was the real-time monitoring of the signal equipment to see that all components were functioning properly.

\subsubsection{Salt Lake City, Utah}

Salt Lake City has taken a very proactive approach to signal system management. However, during the installation of the fiber optic communications backbone, either inadequate materials or workmanship were used. Consequently, the municipality is now having a difficult time adding intersections to the system. The below-standard construction methods have manifested in the lack of communications capability and difficulty in adding new lines to the existing center.

The city employs three persons in the traffic control center to operate the system. These people collect data, perform traffic studies and modify signal plans but do not monitor the system in any regular fashion. There is a combination of city, state, and county agencies involved in an attempt to coordinate the signals to provide seamless integration

\subsubsection{Tucson, Arizona}

Tucson's system is set up mainly to provide for monitoring in the traffic control center. A point of significance is that no persons are required to actually be present at the system during the monitoring process. The system is set to page members of the traffic control team if a signal has raised an alarm. According to the manager, this allows the staffing to be smaller but still provide adequate monitoring of hardware malfunctions. The staffing on the system is comprised of four persons. Two of these persons handle communications issues. These issues include correcting loss of connection to signal 
controllers, replacement of burned out signal bulbs, and the reconfiguration of approaches when detection loops break down. The other two individuals assume the signal management functions which include any break downs in coordination or optimization of signal timings to serve changes in traffic demand.

While the system is not currently used to upgrade signal timings there are some changes in the future for the area. The city has plans to add ITS hardware such as dynamic message signs and surveillance cameras to assist in the management of incidents on area freeways. While there are no signals on the freeways in this area, the existing communications backbone installed with the signal system may be used for additional types of communications.

\subsubsection{Observations}

The municipalities that fall into the medium involvement group interact more with their systems and obtain more functionality than those jurisdictions that fell into the first group. There is still a larger amount of functionality to be gained to from the installations in these locations.

One of the main things to note is that in this group there is very little manpower devoted to the system. In some cases, there is little or no training in the development of traffic plans and signal timing, and in some cases there are no people trained in the maintenance aspects of the system. It is important to note that realizing the maximum potential of such a system requires staffing that is trained in the basics of these aspects.

Another component, which is lacking in the case of several sites, is system training. None of these jurisdictions seem to have much training about the operations and capability of the system in their jurisdiction. Without an awareness of the capabilities of the infrastructure, it will not be possible to operate to its fullest potential.

The final conclusion is a further testament to the importance of planning. This is best demonstrated in the case of Salt Lake City. As a result of changing demands on the installation, the addition of new signals has left the city with a high-cost project each time they wish to expand the signal system beyond its current borders. Issues such as lack of adequate planning, inefficient system training, and untrained staff seem to be limiting agents for this group of municipalities.

\subsection{High Involvement Systems}

Systems that fall into this category devote resources to both coordination and management of the signals, meaning that there is real-time observation of the system and also dynamic intervention with the signal timings. Another way that locations were placed into this category was through the development of inventive ways to integrate or operate the systems. 


\subsubsection{Englewood, Colorado}

Englewood is currently in the process of bringing all of the city's seventy signals into a connected system. At the time of the telephone contact, they had integrated about $85 \%$ of the signals into the system. The system is used for monitoring the controllers, coordinating with other locations, and modifying the timing plans. An interesting aspect of the system design is that in case of a communication failure, it is set to revert to closed loop operations, resulting in an efficient back-up strategy in case of failure.

Two people staff the traffic control center. One is a traffic engineer who oversees the system and generates the traffic plans. The other is a technician who is in charge of correcting hardware failures in the system. A significant aspect of the success of this system was the planning before it was implemented. A consultant was hired to evaluate systems and make recommendations based on the findings. According to the municipality, the consultant's evaluation helped to provide a system that accurately matched the needs of the location to the system that was purchased.

\subsubsection{Charlotte, North Carolina}

Charlotte is currently operating two systems to provide their signal coordination. As with Indianapolis, there is a more robust system in operation to manage traffic in the CBD and on main arterials into and out of the city, with a smaller system running the outlying signal corridors. Both of these systems are used to respond to incidents and monitor the hardware for failure. The outlying system is a closed loop system with a dial-up modem connecting the master to the central on a predetermined schedule. The downtown system is comprised of a centralized system that is in continuous communication with the local controllers.

At the time of the interview, the city was in the process of phasing out the outlying system due to age and lack of support from the system provider. This phase-out was occurring in conjunction with a communications system upgrade. Plans called for fiber optic communication lines to be installed along the corridors, to support not only the signal system but also surveillance cameras and dynamic message signs to help better maintain the traffic flow around the city.

The operations center has five persons on staff comprised of two operators, two signal specialists, and a manager. The operators are tasked with monitoring the system during peak times and making minor adjustments to the signal timing plans. The specialists do most of the timing work and also develop the new signal warrants for the city.

\subsubsection{Mesa, Arizona}

Mesa's coordinated signal system is comprised of ninety-eight intersections connected on a fiber optic backbone. The coordinated signal system is used to provide constant monitoring and timing changes throughout the city. Also, there are special 
timing plans, which are implemented during special events to help better manage the event traffic. The traffic control staff is comprised of three persons, all of whom are signal analysts. It is through the use of this highly trained staff that they are able to implement special timing plans for any special events.

\subsubsection{Baton Rouge, Louisiana}

Baton Rouge's system was found to be one of the most innovative of those researched within this project. Several practices exist within this system that help to increase the utility of the system to the public. First and foremost is the organization of the management staff for this system. Unlike many other municipalities, the city's traffic control branch was under the same manager who handles the city's emergency phone system. In fact, the traffic control and 911 emergency telephone operations centers were located in the same room. This allows for much faster response to any sort of traffic incident which has occurred, allowing both the mobilization of emergency workers to the scene and also the rerouting of traffic through the centralized system. Also, in the cases of emergency vehicles attempting to move around the city it is possible for the traffic control staff to aid in the process by altering the traffic and rerouting vehicles off of clogged arteries.

Another interesting, albeit more common, practice is unique timing plans for special events. This was most evident in the timing plans implemented for Louisiana State University (LSU) home football games. Tiger Stadium has a capacity of 91,600, resulting in a significant amount of traffic on game days. There are special plans set up for each of the visiting teams that the college plays. These are based on which direction the visiting traffic is expected to arrive from. Three people maintain the entire traffic system. One of these individuals is responsible for the timing plans and software issues, one manages the hardware issues, and the third is the manager who maintains the entire facility.

\subsubsection{Orlando, Florida}

Orlando's system is based on a highly scheduled process of signal optimization. There are 408 intersections controlled by this system, with each controller capable of notifying the control center within three minutes of a hardware failure. The current goal of the system operators is to re-time one third of the signals every year. It was noted that the initial goal was to re-time each signal every year but the resources are not available at this time. The traffic center is staffed by eight people, five of whom operate the system, one who does timing plans, one who implements, or makes software adjustments, to the system, and one signal system engineer. This results in very high level of interaction with the system and fast response to signal alarms.

\subsubsection{Seminole County, Florida}

Seminole County's system is a component of a broader system that was funded by a grant from the FHWA. The system controls the counties of Seminole and Orange as 
well as a part of the City of Orlando. The system uses a one-gigabyte per second fiber optic network that provides for a significant amount of communications possibilities. The excess line space is leased to schools and colleges in the area, which helps pay for the costs of the system. However, officials indicated that the system is not constantly monitored and is mainly used for maintenance, emergency vehicle preemption and traffic data collection. The majority of the retiming efforts are subcontracted through the Florida Department of Transportation to consultants. One person is kept on staff to make any changes that need to be made. Plans were also being developed to move the system to the emergency call center, similar to Baton Rouge. However, there are no plans for the system to be monitored on a regular basis.

\subsubsection{Las Vegas, Nevada}

Las Vegas's system is comprised of 500 signals that are located in three different jurisdictions. Operators in each of the jurisdictions communicate information, volumes and timings to the other participants. The cooperation between these facilities is used to help maintain a higher level of coordination throughout the corridors surrounding Las Vegas than would be possible by each system independently. The system was found to be used mainly for signal timing, but plans were under development for the addition of dynamic message signs and cameras along the corridors to aid in incident management. Thirteen people currently staff the traffic center, but a substantial upgrade to more than fifty-five was planned. Part of the reason for this increase was that signals are being consistently added to the system at a rate of three a month. Furthermore, there are plans to expand the system across the state border with Arizona. Also, the city was looking for a professional engineer to take over the management of signal timings. The existing process involved a "roughing in" of the timing plans and the field observation to ensure that the traffic was operating in the desired manner.

\subsubsection{Observations}

It is expected that the key to making a signal management system work at high efficiency is the proper balance between human resources, management practices, and technological resources. All of the locations that were found to operate an efficient system have qualified staff who monitor and develop timings for the signals. In some cases, inventive ideas have helped to further increase the success of these systems. The balance between the needs for traffic signal management and the system (hardware, software, and personnel) to meet these needs is what yields the best results. The next section demonstrates the variability in the balance between technological resources and system size as the relationship between the number of signals managed and the number of workstations in the traffic management center.

\subsection{Signals Managed Per Workstation}

The database that was supplied by the signal vendor was analyzed to determine if there was a discernable relationship between the number of signals managed and the number of workstations in the traffic management center. This is of interest in order to 
attempt to provide some statistical statements that relate number of workstations to operation of traffic signals. The analysis is provided in the appendix, and had a total of 58 observations. Limitations of the analyses of the database include the fact that all of the municipalities were using software provided by the same company. Furthermore, since each system must have at least one workstation to operate, some of the smaller systems in terms of signals managed have biased the data downward. For example, a system that has only five signals requires at least workstation, the same as a system with ten or twenty signals.

The analysis is presented in two parts. First the average rate of workstations required per signal managed was computed, along the maximum and minimum rates, standard deviation, and coefficient of variation. These data will be provided in the following section, along with commentary related to the statistical validity of the relationship and the appropriateness for use. Second, the regression analysis that was performed will be described, along with the equations generated, the corresponding coefficient of regression $\left(\mathrm{R}^{2}\right)$ values, and recommendations for usage. This will be presented in Section 4.4.2.

\subsubsection{Average Rates}

Both the weighted and unweighted average rates of workstations required per signals managed were computed. For the weighted average rate, the sum of all signals managed across all systems were summed and divided by the sum of all workstations. The result was 25.0 signals per workstation. For the unweighted average rate, the number of signals managed was divided by the number of workstations for each individual signal system. The average of these rates was then computed, along with the identification of the maximum and minimum rates, and the computation of the standard deviation and coefficient of variation. The unweighted average was 24.0 signals per workstation, with a maximum and minimum of 300 signals per workstation and 1 signal per workstation, respectively. The standard deviation was 40.7 signals per workstation, which resulted in a coefficient of variation of $169 \%$. Since the coefficient of variation of random database would be $100 \%$ (i.e, mean = standard deviation), the statistical quality of this relationship was highly questionable.

It was noted earlier that the smaller systems might tend to skew the data since even a signal system of one signal requires at least one workstation. Therefore, an additional analysis was performed in which all signal systems having only one workstation were disregarded. The resulting sample size was 32, with an average value of 33.0 signals per workstation. However, the standard deviation increased to 52.3 signals per workstation for a coefficient of variation of $158 \%$. As such, a statistically acceptable average rate could not be found.

\subsubsection{Regression Analysis}

Four types of regression models were formulated using the statistical tools in Microsoft Excel. Each variable was tested as both linear and logarithmic, yielding four 
different combinations. In the regression models, the number of signals was taken as the independent variable, and the number of workstations was the dependent variable. This is the opposite of the form that the average rate was presented. Each regression model is presented along with the coefficient of regression $\left(\mathrm{R}^{2}\right)$.

\section{Linear - Linear}

Number of Workstations $=2.09+0.01$ (Number of Signals); $\mathrm{R}^{2}=0.19$

\section{Logarithmic - Logarithmic}

$\log ($ Number of Workstations $)=-0.22+0.37^{*} \log ($ Number of Signals $) ; \mathrm{R}^{2}=0.49$

\section{Linear - Logarithmic}

Number of Workstations $=-0.58+2.30 * \log ($ Number of Signals $) ; R^{2}=0.37$

$\log \left(\right.$ Number of Workstations) $=0.22+0.001$ (Number of Signals); $R^{2}=0.22$

The regression model form with the highest coefficient of regression value was the log-log form, with a value of 0.49 . Since the coefficient of regression represents the percentage of the variation in the independent variable that is explained by the dependent variable, the conclusion can reached that at best, less than half of the variation in the number of workstations required was attributable to the number of signals managed. Other key variables likely include financial constraints and the size of the staff, neither of which was available to perform further analysis. Thus, none of the regression equations are recommended for usage.

\subsubsection{Observations}

While the relatively large database of signals managed versus workstations in the traffic management center was viewed as a valuable resource when originally obtained, no discernable trends could be gleaned from the data. This is in spite of the fact that all of the municipalities in the database were using signal system software from the same vendor. The variability that would have been encountered had multiple system types been included likely would have been even greater. In some respects, this trend points to the role of other variables, likely largely financial, that come to bear on decisions related to required technological resources. Conversely, the trend also points out the inconsistency in the planning and development of these systems, which in turn illuminates the need for additional guidance in this regard. This is evident in the fact that no discernable trends exist. The lack of trends leads one to determine that the number of installation of workstations is a primarily random event.

\subsection{Staffing}

Table 4.1 provides a summary of the signals integrated into the system and the staff assigned to the system for some of those locations surveyed. These values were used to develop ratios which can be utilized in staff planning by the municipalities. However, before the data are discussed, several locations should be identified for their anomalous conditions. The ratios for San Leandro and Indianapolis are outliers due to 
special circumstances in these locations. In the case of San Leandro, the signals were disconnected due to conditions beyond their control. Also, it should be noted that many of these system are in transient conditions. In the case of Indianapolis, special experience and training exist that allows for an exception to the values determined above. The final operating conditions have not been reached and is therefore possible for the numerical values to be skewed at locations that are either adding more signals or looking for new employees.

Table 4.1 Staffing Conditions of Study Facilities

\begin{tabular}{|c|c|c|}
\hline Site & $\begin{array}{l}\text { Percent of Signals on the } \\
\text { System }\end{array}$ & $\begin{array}{c}\text { Ratio of System Signals per } \\
\text { Staff Member }\end{array}$ \\
\hline \multicolumn{3}{|l|}{$\begin{array}{l}\text { Low Involvement } \\
\text { Municipalities }\end{array}$} \\
\hline Redwood City & $75 \%$ & 45 \\
\hline San Leandro & $7 \%$ & 1 \\
\hline \multicolumn{3}{|l|}{$\begin{array}{l}\text { Medium Involvement } \\
\text { Municipalities }\end{array}$} \\
\hline Indianapolis & $\begin{array}{l}22 \% \\
55 \%\end{array}$ & 770 \\
\hline Santa Clara & $95 \%$ & 58 \\
\hline Beaumont & $45 \%$ & 15 \\
\hline Salt Lake City & $91 \%$ & 183 \\
\hline Tucson & $100 \%$ & 120 \\
\hline \multicolumn{3}{|l|}{$\begin{array}{l}\text { High Involvement } \\
\text { Municipalities }\end{array}$} \\
\hline Englewood & $82 \%$ & 20 \\
\hline Charlotte & $\begin{array}{l}23 \% \\
53 \%\end{array}$ & 93 \\
\hline Mesa & $31 \%$ & 32 \\
\hline Baton Rouge & $90 \%$ & 166 \\
\hline Orlando & $93 \%$ & 51 \\
\hline Seminole County & $100 \%$ & 27 \\
\hline Las Vegas & $100 \%$ & 38 \\
\hline
\end{tabular}

There are two trends that can be identified from this data. If a location desires to operate within the Medium Involvement Systems group, a range of 75 to 125 signals per staff person is a reasonable condition at which to operate. For locations that desire to operate within the range of the High Involvement Systems grouping, 30 to 55 signals per person is the staffing condition which should satisfy this requirement. However, note that the addition of highly trained specialized staff such as signal or traffic engineers will allow the ratio of signals per person to rise while maintaining a consistent level of operation.

Also noted was the fact that the ratio of signals per workstation in the traffic management center was approximately 25. However, the statistical analysis of this 
database revealed that the relationship is weak, and illustrated the fact that the number of signals was not necessarily a major factor in the sizing of technological resources for the traffic management center.

\subsection{Research and Planning}

The first and most fundamental component of the optimum operation of these systems is planning. At the outset of a system installation project, some goals need to be developed which will shape the final result of the system. A municipality that is planning to implement a traffic signal system needs to clearly define the final operational conditions. If the goal is to have the system operate with either low or medium involvement from their staff, then a municipality can determine the amount of data which will need to travel across the communications backbone of the system. Also, before any purchases are made, it should be determined if the desire for more complex control exists in the future. The above-mentioned decisions will then shape the communications structure that must be put into place. In the case of a small amount of information traversing the network, it will be possible for the municipality to save resources. These savings will manifest themselves in not purchasing system components and infrastructure which will not be needed for the installation. In the case of a robust data stream (one including traffic data, multiple timing plans, and other information), the municipality will find that a slower speed of signal integration must be taken in order to achieve a high quality installation or a slower rate of dollar investment.

Secondly, the municipality will be able to make a decision relating to the system vendor. By understanding the range of user functions that are included in each control package and identifying only those components which will be used, the municipality can reduce the resources invested in the system. It may be that the base software package for a coordinated signal system will provide the municipality with the components that will be utilized, making further software expansions unnecessary.

The third major component which should be determined in the research and planning stage is the pace at which signals are integrated into the system, and a priority ranking (based on the locations need) to guide the integration. In bringing a large system online, there are many problems that may occur. It would be advisable to slowly integrate corridors. By determining several key corridors with which to begin the operation, it will be possible to modify the structure of the system after some valuable information has been obtained. The slow integration will also provide the system operators with the opportunity to learn the system as it grows instead of trying to operate a large robust system with little or no experience.

\subsection{Location and Layout}

Having an understanding of the implementation of the system, it will be easier to determine the corridors that will eventually be integrated into the system. It should be understood that this plan is not a concrete operation structure, but instead is a flexible plan that is capable of addressing future growth or changes in traffic patterns. However, 
with this plan it will be easier for the municipality to locate the control center to minimize the communication infrastructure.

If the municipality plans to implement emergency preemption or incident management, this would also be time to integrate this system with the local emergency 911 center in order to maximize the benefits gained from the system in these areas.

Examining topographical conditions along the corridor will help to give the developers a better idea of the investments that will have to be made in the communications structure, thus allowing the pace of expansion and implementation to increase.

\subsection{Concluding Remarks}

Conclusions which can be obtained from this data are that, with preliminary research and planning, many municipalities would have a system which is more in line with the operations desired by the municipalities. Additionally, by considering the location of the signal corridors in relation to the center of control the municipalities would save financial resources which would have been invested in the communications infrastructure. The preceding chapter presented and investigated the data collected as part of this research endeavor. Chapter 5 then synthesizes these results, and discusses the limitations of the research and ideas for future research in the area.

In summation, the following lessons can be learned from those locations surveyed:

- Planning is very important no matter what level of involvement a municipality is looking to invest into the system.

- System variables such as staffing, communications backbone type, future goals of the system, and intersection adjustment schedules should be decided prior to the implementation an interconnected traffic signal system.

- The communication backbone is what makes one of these systems different from a standard signalized intersection. Without this link any advantages gained from such a system are negated.

- Prepare to have a staff trained for the goals of the system. Without the proper personnel, it may not be possible to achieve the system goals. This training refers to the skill set which is best suited to the manner in which the installation is to be operated. 


\section{CHAPTER 5 - CONCLUSIONS AND RECOMMENDATIONS}

\subsection{Introduction}

As demonstrated in Chapter 4, there are several key components which must be coordinated in order to reach the desired operation of a traffic signal system. These components are research and planning, location and layout, and the human and technological resources. Also discussed in Chapter 5 are some of the limitations of this study along with suggestions for future research.

\subsection{Conclusions}

- Planning is very important no matter what level of involvement a municipality is looking to invest into the system. With proper planning it will be possible to maximize the benefit for each dollar spent on the installation and the jurisdiction can also develop a clear set of goals for their installation. By examining the entire system it will be possible for the municipality to determine what level of involvement is best suited to the location

- System variables such as staffing, communications backbone type, future goals of the system, and intersection adjustment schedules should be decided upon prior to the implementation of an interconnected traffic signal system. These decisions will help determine the schedule at which intersections are brought online. Any changes made once the system begins to operate can be very costly. By determining these components prior to the implementation of the system a municipality can maximize their resources. Once the above variables have been determined, the municipality will have an idea of the speed and volume at which signals can be added to the network. These decisions will also clarify the number of signals which may feasibly be added to the system. This information will provide the municipality a clear vision of the final operating condition and the expected completion date.

- Be prepared with a backup plans for communication. The communication backbone is what makes one of these systems different from a standard signalized intersection. Without this link, any advantages gained from such a system are negated. Other system variables such as the future desires of the system may require additional consideration to be made in this respect.

- Prepare to have a staff trained for the goals of the system. Without the proper personnel, it may not be possible to achieve the system goals. This training refers to the skill set which is best suited to the installation to be operated. By using the information provided in Table 4.1, it will be possible for the municipalities to make some informed decisions about the type of staffing numbers which will be needed to operate at the level of involvement that is desired. 
By following the above conclusions, a municipality is on track to obtain what is expected from the investments made. Through careful consideration of each of these conclusions a municipality will be better prepared to install and operate a coordinated traffic control system to meet the goals set forth by the jurisdiction.

\subsection{Limitations of the Research}

There are two of the main limitations to this research. First, the phone surveys and data collection were conducted in the years 2001 and 2002. With the continuous evolution of the subject systems discussed in this research, it is likely that many changed soon after completion of the data collection. Second, many of the conclusions drawn from this research are based on data that is largely the perception of respondents to the phone survey. This was a necessity, as budgetary and time limitations restricted the ability of the researchers to investigate these systems first-hand. Due to the above mentioned limitations there are several bias that resulted in the data set. First, because it is the larger municipalities which have the more robust systems, there is a bias toward larger municipalities. Also, in part due to the bias toward larger locations, the ability to maintain staff and equipment is also sampled on the higher end of the spectrum. However, since most of the conclusions drawn are consistent with the principles of good engineering planning and design, and stand up to the test of common sense, it is likely that the study was not negatively impacted as a result.

\subsection{Ideas for Future Research}

The area of signal systems is a quickly growing and expanding area. Advances in both computer and data transmission technology will impact designs for system configuration in the future. These phenomena, in combination with the NTCIP standards for traffic signal systems, will afford the opportunity for vastly different trends to appear in this field. More research is recommended in this area with a larger study group and possibly more precisely defined groupings. In the process of taking a larger sample it will also be possible to develop a more formal reproducible phone survey. Completing the same study with these larger survey samples will help to better define some of the variables. Additionally, field visits to some of the many centralized signal systems would provide for a more uniform analysis of each location.

Future research that will provide useful information is to contact the municipalities surveyed in this study to see if any changes were made to the system, and if changes were made, how they impacted the system. Also, information on changes in the local environment should be collected. From this, it would be possible to better determine what changes are beneficial to these systems.

Furthermore, a case study starting from the early planning of a system and following the complete installation and operation would provide some concrete evidence of the value of the ideas set forth in this research. This study would provide for clear determinations to be made on planning decisions. 
Finally, a study should be conducted on the different software packages for signal control systems. This study would be able to clearly define the conditions in which certain systems should be used. Such a resource would be very useful to governing agencies at the planning stage. 


\section{References}

Adams, Donald D; and Huffine, Charles W; “Safety Benefits of Signal Coordination”; ITE Compendium of Technical Papers; 1995, p219-222

Belrose, William M, et al.; Addition of Adaptive Control to the Minneapolis Computerized Traffic Signal System; ITE Compendium of Technical Papers; 1996, p208-212

Federal Highway Administration, Rural ITS Toolbox, November 2001

Federal Highway Administration (FHWA): NTCIP 9001(Draft) National Transportation Communications for ITS Protocol (NTCIP) Guide June 2001

Garber, Nicholas J.; and Hoel, Lester A; Traffic and Highway Engineering, $2^{\text {nd }}$ ed, PWS Publishing, New York, 1999

Gordon, Robert L; Reiss, Robert A.; Haenel, Herman; Case, E. Ryerson; French, Robert L.; Mohaddes, Abbas; and Wolcott, Ronald; Traffic Contol Systems Handbook, United States Department of Transportation, Federal Highway Administration, Report number FHWA-SA-95-032, Washington D.C., 1996

Kraft, Walter D; Traffic Signal Control Systems Maintenance Management Practices NCHRP Synthesis 245, 1997

Lui, Henry X et al.: Adaptive Signal Control System with On-Line Performance Measure: TRB Paper \# 02-3353; 2002

Mohammadish, Mehdi, et al.; Signal Timing Optimization-Bloomfield Avenue Closed Loop Traffic Signal System Case Study; ITE Compendium of Technical Papers; 1995, p169-173

National Electronics Manufacturers Association (NEMA); NEMA TS 3.5-1996 November 1997

Skehan, Sean; “Adaptive Traffic Control Systems”; ITE Compendium of Technical Papers; 1996, p203-207

United States Census Bureau; http://www.census.gov; 2000

White, Curt M; Data Communications and Computer Networks $2^{\text {nd }}$ ed, PWS Publishing, New York, 2002 


\section{Appendices}




\section{Appendix A - Phone Interview Question List}

1) What brand of system is operated in your location?

2) How long has this system been in operation?

3) How many signals are currently connected to the system?

4) How many signals exist in your jurisdiction?

5) What is the population of the area?

6) What is the layout configuration of your installation? (e.g. master/slave or direct connect)

7) How do individual intersections communicate?

8) What system capabilities do you utilize at your location?

9) Do you have a traffic control center?

10) How does your traffic control center communicate with field installations?

11) How many workstations are used to operate/interact your system?

12) How are these workstations utilized?

13) How many people work in the traffic control center?

14) What are their job tasks?

15) What are their job titles?

16) What are their qualifications?

17) How does the staff interact with the traffic control system?

18) Viewing this discussion is there anything that you would like to add to the things discussed?

19) Are you aware of any other coordinated signal systems in other municipalities? 


\section{Appendix B Municipality Database Statistical Analysis}

\begin{tabular}{|c|c|c|}
\hline Signals & Workstations & Rate \\
\hline 25 & 1 & 25 \\
\hline 130 & 8 & 16.25 \\
\hline 200 & 4 & 50 \\
\hline 50 & 1 & 50 \\
\hline 5 & 1 & 5 \\
\hline 100 & 2 & 50 \\
\hline 60 & 2 & 30 \\
\hline 100 & 2 & 50 \\
\hline 30 & 1 & 30 \\
\hline 150 & 12 & 12.5 \\
\hline 30 & 1 & 30 \\
\hline 400 & 5 & 80 \\
\hline 5 & 1 & 5 \\
\hline 5 & 1 & 5 \\
\hline 5 & 1 & 5 \\
\hline 10 & 1 & 10 \\
\hline 10 & 1 & 10 \\
\hline 5 & 1 & 5 \\
\hline 35 & 1 & 35 \\
\hline 60 & 8 & 7.5 \\
\hline 5 & 1 & 5 \\
\hline 10 & 1 & 10 \\
\hline 125 & 6 & 20.83333 \\
\hline 29 & 5 & 5.8 \\
\hline 90 & 6 & 15 \\
\hline 70 & 3 & 23.33333 \\
\hline 60 & 3 & 20 \\
\hline 6 & 3 & 2 \\
\hline 20 & 1 & 20 \\
\hline
\end{tabular}

\begin{tabular}{|c|c|c|}
\hline Signals & Workstations & Rate \\
\hline 40 & 5 & 8 \\
\hline 30 & 3 & 10 \\
\hline 40 & 3 & 13.33333 \\
\hline 600 & 2 & 300 \\
\hline 12 & 1 & 12 \\
\hline 40 & 2 & 20 \\
\hline 400 & 8 & 50 \\
\hline 300 & 6 & 50 \\
\hline 15 & 3 & 5 \\
\hline 70 & 4 & 17.5 \\
\hline 40 & 4 & 10 \\
\hline 15 & 1 & 15 \\
\hline 7 & 1 & 7 \\
\hline 4 & 1 & 4 \\
\hline 5 & 1 & 5 \\
\hline 40 & 4 & 10 \\
\hline 40 & 4 & 10 \\
\hline 40 & 2 & 20 \\
\hline 10 & 1 & 10 \\
\hline 44 & 2 & 22 \\
\hline 75 & 3 & 25 \\
\hline 20 & 4 & 5 \\
\hline 1 & 1 & 1 \\
\hline 1 & 1 & 1 \\
\hline 100 & 2 & 50 \\
\hline 100 & 2 & 50 \\
\hline 5 & 1 & 5 \\
\hline 10 & 1 & 10 \\
\hline 15 & 1 & 15 \\
\hline
\end{tabular}

\begin{tabular}{|l|r|r|r|}
\cline { 2 - 4 } \multicolumn{1}{c|}{} & \multicolumn{1}{c|}{ Signals } & Workstations & \multicolumn{1}{c|}{ Rate } \\
\hline sum & 3949 & 158 & \\
\hline ave rate & 25.0 & & 24.0 \\
\hline $\max$ & & & 300.0 \\
\hline $\min$ & & & 1.0 \\
\hline stdev & & & 40.7 \\
\hline & & & $169 \%$ \\
\hline
\end{tabular}


Linear Regression

SUMMARY OUTPUT

\begin{tabular}{lc}
\hline Regression Statistics & \\
\hline Multiple R & 0.438585162 \\
R Square & 0.192356945 \\
Adjusted R Square & 0.177934747 \\
Standard Error & 2.113034168 \\
Observations & 58
\end{tabular}

ANOVA

\begin{tabular}{|c|c|c|c|c|c|c|c|c|}
\hline & $d f$ & SS & $M S$ & $F$ & Significance $F$ & & & \\
\hline Regression & 1 & 59.55105685 & 59.55106 & 13.33756147 & 0.000573794 & & & \\
\hline Residual & 56 & 250.03515 & 4.464913 & & & & & \\
\hline Total & 57 & 309.5862069 & & & & & & \\
\hline & Coefficients & Standard Error & $t$ Stat & $P$-value & Lower 95\% & Upper 95\% & Lower $95.0 \%$ & Upper $95.0 \%$ \\
\hline Intercept & 2.09 & 0.326816203 & 6.405443 & 3.30405E-08 & 1.438711568 & 2.748093749 & 1.438711568 & 2.748093749 \\
\hline X Variable 1 & 0.01 & 0.002536587 & 3.652063 & 0.000573794 & 0.004182384 & 0.014345166 & 0.004182384 & 0.014345166 \\
\hline
\end{tabular}

Number of Workstations $=2.09+0.01$ (Number of Signals)

$\mathrm{R}$ Square $=0.19$ 
Log-Log regression SUMMARY OUTPUT

\begin{tabular}{lr}
\hline \multicolumn{2}{c}{ Regression Statistics } \\
\hline Multiple R & 0.69908603 \\
R Square & 0.488721278 \\
Adjusted R Square & 0.479591301 \\
Standard Error & 0.23336252 \\
Observations & 58 \\
\hline
\end{tabular}

ANOVA

\begin{tabular}{lccccc}
\hline & $d f$ & $S S$ & $M S$ & $F$ & Significance $F$ \\
\hline Regression & 1 & 2.915102078 & 2.915102 & 53.52929897 & $1.03818 \mathrm{E}-09$ \\
Residual & 56 & 3.049651677 & 0.054458 & & \\
Total & 57 & 5.964753755 & & & \\
\hline
\end{tabular}

\begin{tabular}{|c|c|c|c|c|c|c|c|c|}
\hline & Coefficients & Standard Error & $t$ Stat & $P$-value & Lower 95\% & Upper 95\% & Lower $95.0 \%$ & Upper 95.0\% \\
\hline Intercept & -0.217593219 & 0.078137579 & -2.784745 & 0.007294643 & -0.374121495 & -0.061064943 & -0.374121495 & -0.061064943 \\
\hline X Variable 1 & 0.365637871 & 0.049975298 & 7.316372 & 1.03818E-09 & 0.265525385 & 0.465750356 & 0.265525385 & 0.465750356 \\
\hline
\end{tabular}

$\log \left(\right.$ Number of Workstations) $=-0.22+0.37^{*} \log ($ Number of Signals)

$\mathrm{R}$ Square $=0.49$ 
Workstations Linear - Signals Log SUMMARY OUTPUT

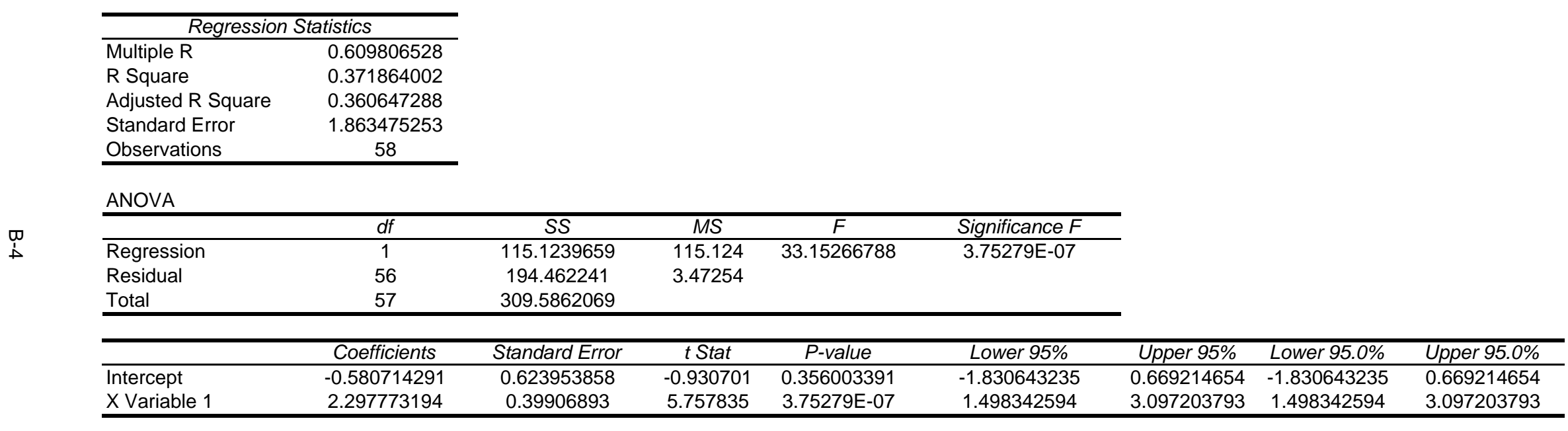

Number of Workstations $=-0.58+2.30 * \log ($ Number of Signals $)$

$\mathrm{R}$ Square $=0.37$ 
Workstations Log - Signals Linear SUMMARY OUTPUT

\begin{tabular}{lc}
\hline \multicolumn{2}{c}{ Regression Statistics } \\
\hline Multiple R & 0.46709161 \\
R Square & 0.218174572 \\
Adjusted R Square & 0.204213404 \\
Standard Error & 0.288573962 \\
Observations & 58 \\
\hline
\end{tabular}

\section{ANOVA}

\begin{tabular}{lccccc}
\hline & $d f$ & $S S$ & $M S$ & $F$ & Significance $F$ \\
\hline Regression & 1 & 1.301357599 & 1.301358 & 15.62724314 & 0.000218728 \\
Residual & 56 & 4.663396155 & 0.083275 & & \\
Total & 57 & 5.964753755 & & & \\
\hline
\end{tabular}

Total

Coefficients

\begin{tabular}{lc}
\hline & Coefficients \\
\hline Intercept & 0.215058501
\end{tabular}

Standard Error

t Stat

$P$-value

Lower 95\%

Upper 95\% Lower 95.0\%

0.125648304

Upper $95.0 \%$

$\mathrm{X}$ Variable 1

0.001369435

0.044632807
0.000346418

3.953131

1.14352E-05

0.125648304

0.304468698

0.000675477

0.304468698

$\log ($ Number of Workstations) $=0.22+0.001$ (Number of Signals)

$\mathrm{R}$ Square $=0.22$ 